\title{
TITLE:
}

\section{Phase transitions of binary alloys with elastic inhomogeneity}

\section{$\operatorname{AUTHOR}(S)$ :}

Onuki, A; Furukawa, A

\section{CITATION:}

Onuki, A ...[et al]. Phase transitions of binary alloys with elastic inhomogeneity. Physical Review Letters 2001, 86(3): 452-455

\section{ISSUE DATE:}

2001-01-15

URL:

http://hdl.handle.net/2433/49917

\section{RIGHT:}

Copyright 2001 American Physical Society 


\title{
Phase Transitions of Binary Alloys with Elastic Inhomogeneity
}

\author{
Akira Onuki and Akira Furukawa \\ Department of Physics, Kyoto University, Kyoto 606-8502, Japan
}

(Received 13 June 2000)

\begin{abstract}
In elastically inhomogeneous alloys, in which the shear modulus depends on the composition, coarsening in phase separation can be pinned even without quenched disorder. Here networks in the soft phase enclose the domains in the hard phase. Highly asymmetric strains in the two regions create large free energy barriers that prevent further coarsening. Thus a phase transition occurs between the one-phase and the glassy two-phase states. We numerically obtain the phase diagram and show that this transition is discontinuous at any composition. Therefore there is no critical point.
\end{abstract}

DOI: $10.1103 /$ PhysRevLett.86.452

In phase separation of alloys, elastic strains resulting from a lattice misfit between the two phases can radically influence the domain morphology [1,2]. As a first approach Cahn presented a Ginzburg-Landau theory in the simplest case of isotropic elasticity with constant elastic moduli [3], where the coexistence curve was shown to be shifted by a constant temperature after elimination of the elastic field from the mechanical equilibrium condition. In the next work on cubic crystals with constant elastic moduli, he derived a dipolar interaction bilinear with respect to the composition fluctuations [4]. Other authors subsequently presented more refined or generalized derivations of the dipolar interaction for anisotropic elasticity [2]. This interaction is long ranged and angle dependent, so it is minimized for particular shapes and configurations of precipitates, consistently explaining a number of experiments $[5,6]$.

Another important ingredient affecting the domain morphology is the difference in the elastic moduli between the two phases. It has often been observed that harder cuboids with a larger shear modulus $C_{44}$ are wrapped by a softer matrix. A typical example is given by $\mathrm{Ni}_{4} \mathrm{Mo}$ particles in Ni-16.3Mo alloys [7], where the mean domain size $\bar{r}(t)$ initially increased with increasing aging time but the coarsening virtually stopped with prolonged aging. Such abnormal slowing down occurs for high solute contents under strong elastic constraints, while the usual growth law $\bar{r}(t) \propto t^{1 / 3}$ has been observed in many cases for small volume fractions of precipitates and/or relatively short aging times [7-10]. One of the present authors presented a Ginzburg-Landau theory assuming compositiondependent elastic moduli or elastic inhomogeneity [11]. The resultant dynamic equations can easily be integrated on the computer [12-14], yielding simulation results in agreement with the above experiments. Along the same line Sagui et al. have recently performed large scale simulations of phase separation with elastic inhomogeneity to find a variety of two-phase structures in two and three dimensions [15].

However, it has not been well understood how the phase transition takes place in the presence of elastic inhomo-
PACS numbers: 64.75. $+\mathrm{g}$, 64.70.Kb, 81.30.Bx, 81.30.Mh

geneity. We start with the free energy density,

$$
\begin{aligned}
F=\int d \boldsymbol{r}[ & f_{0}(\psi)+\frac{k_{B} T}{2}|\nabla \psi|^{2} \\
& \left.+\alpha \psi \nabla \cdot \boldsymbol{u}+f_{\mathrm{el}}(\boldsymbol{u})\right],
\end{aligned}
$$

where $\psi\left(\propto c-c_{c}\right)$ is the appropriately scaled composition (measured from a critical value) and $\boldsymbol{u}$ is the elastic field coupled to $\psi$ via the Vegard law. For simplicity we assume that the elastic energy density $f_{\mathrm{el}}(\boldsymbol{u})$ is characterized by a constant bulk modulus $K_{0}$ and a compositiondependent shear modulus given by

$$
\mu=\mu_{0}+\mu_{1} \psi,
$$

within isotropic elasticity. If we assume the mechanical equilibrium condition $\delta F / \delta u_{i}=0$, we obtain $\boldsymbol{u}=$ $-\left(\alpha / L_{0}\right) \nabla w+O\left(\mu_{1}\right)$, where $L_{0}=K_{0}+(2-2 / d) \mu_{0}$ is the longitudinal modulus, and $w$ is determined by

$$
\nabla^{2} w=\psi-M .
$$

The average order parameter $\langle\psi\rangle$ is written as $M$. No externally applied anisotropic strain is assumed. Elimination of $\boldsymbol{u}$ yields the free energy of $\psi$ in the Landau expansion,

$$
\frac{F}{k_{B} T}=\int d \boldsymbol{r}\left[\frac{\tau}{2} \psi^{2}+\frac{u_{0}}{4} \psi^{4}+\frac{1}{2}|\nabla \psi|^{2}+g_{E} \psi Q\right],
$$

where

$$
Q=\sum_{i j}\left(\nabla_{i} \nabla_{j} w-\frac{1}{d} \delta_{i j} \nabla^{2} w\right)^{2} .
$$

Here $\tau=a_{0}\left(T-T_{c}\right)$ is a (scaled) reduced temperature, $T_{c}$ being the so-called coherent critical temperature [3], $g_{E}=\mu_{1} \alpha^{2} / k_{B} T L_{0}^{2}, \nabla_{i}=\partial / \partial x_{i}$, and $d$ is the space dimensionality. The last term in Eq. (4) arises from the composition dependence of $\mu$ and is linear with respect to $\mu_{1}$. It consists of the bilinear term $(1-1 / d) g_{E} M(\delta \psi)^{2}$, because $\int d \boldsymbol{r} Q=(1-1 / d) \int d \boldsymbol{r}(\delta \psi)^{2}$, and the third order term $g_{E} \delta \psi Q$. Picking up the bilinear order terms 
in Eq. (4) we obtain the spinodal curve of the one-phase states,

$$
\tau+3 u_{0} M^{2}+(2-2 / d) g_{E} M=0 .
$$

However, the third order term is a highly nontrivial, nonlocal interaction. It is relevant in the sense that it becomes increasingly important for small $\tau$ and $M$. In fact, the ratio of this third order term to the first bilinear term $(\propto \tau)$ in Eq. (4) is represented by the dimensionless strength,

$$
g_{E}^{*}=g_{E} /\left|u_{0} \tau\right|^{1 / 2},
$$

where we set $\psi \sim\left|\tau / u_{0}\right|^{1 / 2}$. If $g_{E}^{*} \geqslant 1$, we are in a new regime of strong elastic inhomogeneity, where even the thermal fluctuations on the scale of the correlation length are distinctly soft or hard. In terms of observable quantities, this condition is also expressed as $v_{0} \eta^{2}\left|\mu_{1}\right| / k_{B} T \gtrsim$ $\left|T / T_{c}-1\right|^{1 / 2}$. Here $v_{0}$ is the volume of a unit cell and $\eta=(d a / d c) / a\left(\propto \alpha / K_{0}\right)$ is the lattice expansion parameter with $a=a(c)$ being the alloy lattice constant, where the coefficients in the free energy are estimated in the Bragg-Williams theory. Alternatively, we may introduce a characteristic reduced temperature and average order parameter by

$$
\tau_{E}=g_{E}^{2} / u_{0}, \quad M_{E}=g_{E} / u_{0} .
$$

In the strongly inhomogeneous regime we require $|\tau|<\tau_{E}$ and $|M|<M_{E}$. The solid is in the regime of weak elastic inhomogeneity for $|M| \gg M_{E}$ even at $\tau=0$.

We numerically solve the diffusive equation,

$$
\frac{\partial}{\partial t} \psi=\lambda_{0} \nabla^{2} \frac{\delta}{\delta \psi} \frac{F}{k_{B} T},
$$

in two dimensions without thermal noise. In previous numerical work we studied only the weakly inhomogeneous case $\left(\tau<0\right.$ and $\left.g_{E}^{*} \sim 0.1[13]\right)$. There the initial domain growth is isotropic and obeys the Lifshitz-Slyozov law $R(t) \propto t^{1 / 3}$, but in very late stages the elastic inhomogeneity gives rise to asymmetry between the two phases, resulting in extreme slowing down of the coarsening. The pinning occurs when the surface energy $\left(\sim \sigma R^{d-1}, \sigma\right.$ being the surface tension) and the elastic inhomogeneity free energy $\left[\sim k_{B} T g_{E}(\Delta \psi)^{3} R^{d}\right.$ ] per domain are of the same order. Then the characteristic size $R_{E}$ of domains is given by

$$
R_{E}=\sigma /\left|k_{B} T g_{E}(\Delta \psi)^{3}\right| \sim u_{0}^{1 / 2} / g_{E} \sim \xi / g_{E}^{*} .
$$

We note that $\xi=|\tau|^{-1 / 2}$ and $R_{E}$ are the two characteristic lengths involved in the free energy (4). We then consider the free energy barrier per domain preventing coarsening. It is estimated as

$$
(\Delta F)_{E} \sim \sigma R_{E}^{d-1} \sim k_{B} T\left[|\tau|^{2-d / 2} / u_{0}\right] /\left(g_{E}^{*}\right)^{d-1} .
$$

As long as $g_{E}^{*} \lesssim 1$, we have $(\Delta F)_{E} \gg k_{B} T$ for $|\tau|^{2-d / 2} \gg u_{0}$ (the Ginzburg criterion) where the mean field theory holds. This means that pinning should occur even if the thermal noise is included in the simulation.
Although not attained in numerical work, we believe that true equilibrium two-phase states are periodic in space, in which dropletlike hard domains are wrapped by percolating soft regions.

In this Letter we show that pinning occurs rapidly in the strongly inhomogeneous case. Figure 1 shows the inverse perimeter length for quenches with various $g_{E}^{*}$ at $M=0$. The inset indicates that the domain size in pinned states is roughly inversely proportional to $g_{E}^{*}$. Thus, in the strongly inhomogeneous case $g_{E}^{*} \gtrsim 1$, phase transitions occur between one-phase states and pinned two-phase states without much growth of the domains. In Fig. 2 we plot the free energy density difference $\Delta f=F / V-\tau M^{2} / 2-$ $u_{0} M^{4} / 4$ relative to the value in the homogeneous phase as a function of $\tau / \tau_{E}$ at $M / M_{E}=-0.21$, which is close to the maximum point $M / M_{E}=-1 / 6$ of the spinodal curve (6) in two dimensions. The two-phase states with $\Delta f<0$ should be stable against thermal agitations in the presence of the thermal noise. Hence the point at which $\Delta f=0$ on the two-phase branch may be treated as a first order phase transition point. At $M / M_{E}=-0.21$, the transition point thus determined is $0.265 \tau_{E}$, while the spinodal points are $0.53 \tau_{E}$ for the two-phase states and $-0.89 \tau_{E}$ for the one-phase states. As shown in Fig. 3, this hysteretic behavior persists at any $M$. Therefore, no critical point exists under elastic inhomogeneity. Around this first order phase transition we have $\tau \sim \tau_{E}, \psi \sim M_{E}$, and $\xi \sim$ $\tau_{E}^{-1 / 2} \sim R_{E}$. Then the free energy barrier per domain is estimated as

$$
(\Delta F)_{E} \sim k_{B} T\left[\tau_{E}^{2-d / 2} / u_{0}\right] \gg k_{B} T
$$

in the mean field regime. In the asymptotic critical region, if it can be reached, the barrier is weakened, suggesting appearance of periodic two-phase states in equilibrium.

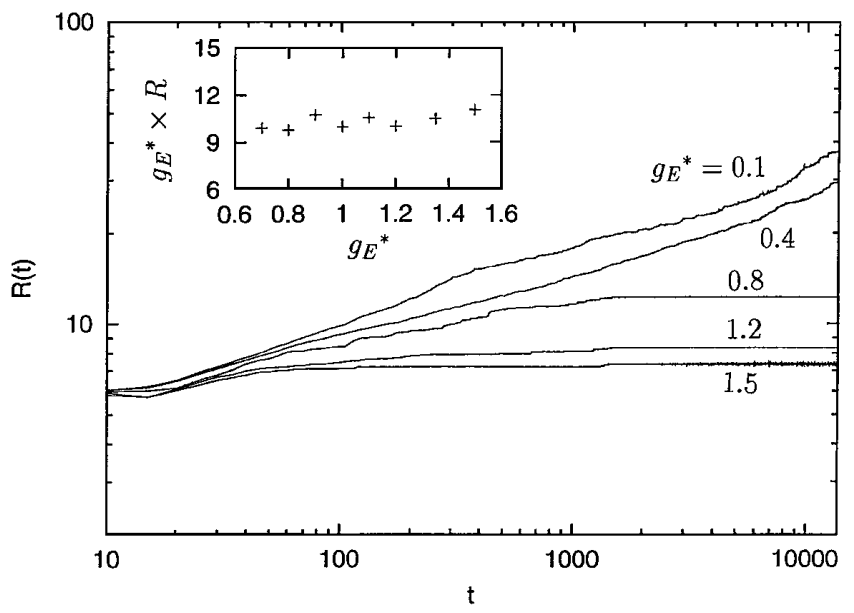

FIG. 1. The domain size $R(t)$ obtained as the inverse of the perimeter length density (in units of $\xi$ ) vs time (in units of $\left.1 / \lambda_{0} \tau^{2}\right)$ at $M=0$ for various $g_{E}^{*}$ within isotropic elasticity in a $248 \times 248$ system. Pinning occurs at early times for larger $g_{E}^{*}$. In the inset the relation $R \propto 1 / g_{E}^{*}$ is shown to hold in pinned states. 


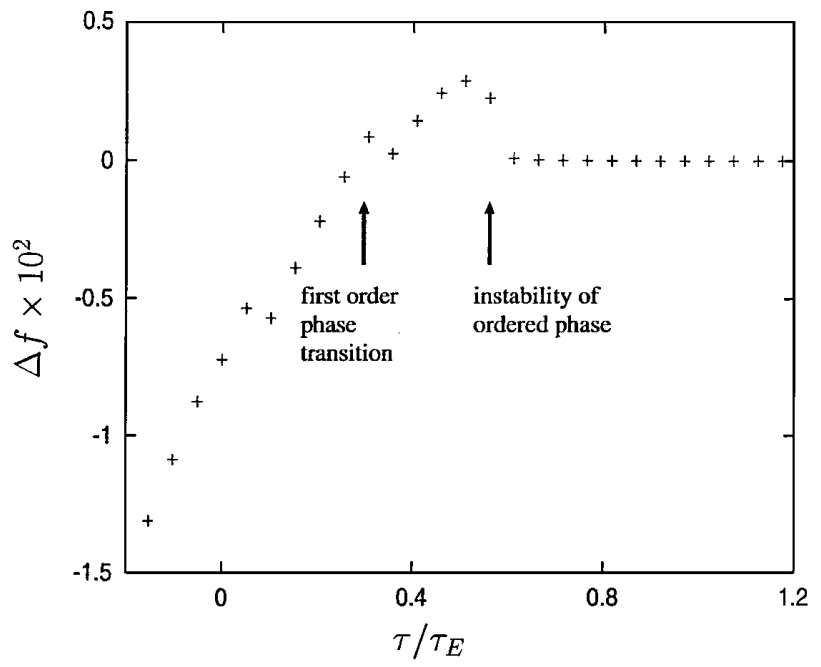

FIG. 2. The difference in the free energy density between onephase and pinned two-phase states (in units of $k_{B} T g_{E}^{4} / u_{0}^{3}$ ) as a function of $\tau / \tau_{E}$ at $M / M_{E}=-0.21$. It vanishes at $\tau / \tau_{E}=$ 0.265 on the two-phase branch, where a first order phase transition is expected. The two-phase states are unstable for $\tau / \tau_{E}>$ 0.53 , while the one-phase states are unstable for $\tau / \tau_{E}<-0.89$ as predicted by Eq. (6) (not shown here).

Figure 3 also shows that the soft regions form a thin network at relatively small volume fractions of the soft component. For such domain structures the space dependence is mostly along the interface normal $\boldsymbol{n}$ except for the junction regions. Then we may set $\nabla_{i} \nabla_{j} w \cong n_{i} n_{j}(\psi-M)$ to obtain the approximate free energy density,

$$
\frac{1}{k_{B} T} f_{\mathrm{eff}}=\frac{1}{2} \tau \psi^{2}+\frac{1}{4} u_{0} \psi^{4}+\bar{g}_{E} \psi(\psi-M)^{2},
$$

where $\bar{g}_{E}=(1-1 / d) g_{E}$. For this free energy density phase separation can occur for

$$
\tau_{\text {eff }}=\tau-4 M \bar{g}_{E}-3 \bar{g}_{E}^{2} / u_{0}<0,
$$

and the interface thickness is given by $\xi=\left|\tau_{\text {eff }}\right|^{-1 / 2}$. In the resultant two phases we have $\psi=\psi_{+}$and $\psi_{-}$with $\psi_{ \pm}=-\bar{g}_{E} / u_{0} \pm\left|\tau_{\text {eff }} / u_{0}\right|^{1 / 2}$, so that

$$
M=-\bar{g}_{E} / u_{0}+\left|\tau_{\text {eff }} / u_{0}\right|^{1 / 2}\left(1-2 \phi_{s}\right),
$$

where $\phi_{s}$ is the volume fraction of the soft regions. The network at small $\phi_{s}$ should dissolve when the layer thickness becomes of the order of $\xi$. However, we cannot determine the network mesh size $\ell_{\text {net }}$ from the quasi-1D free energy density (13) only. In our simulation $\ell_{\text {net }}$ is about 10 times longer than $\xi$ on the points of first order phase transition at small $\phi_{s}$ in Fig. 3. Indeed, these points are nearly on the theoretical curve of $\phi_{s}=0.1$ in Eq. (15).

Next we consider cubic solids characterized by three elastic moduli, $C_{11}, C_{12}$, and $C_{44}$. The degree of anisotropy is represented by $\xi_{a}=\left(C_{11}-C_{12}-2 C_{44}\right) / C_{44}$. In the simplest case of weak elastic anisotropy $\left|\xi_{a}\right| \ll 1$, the

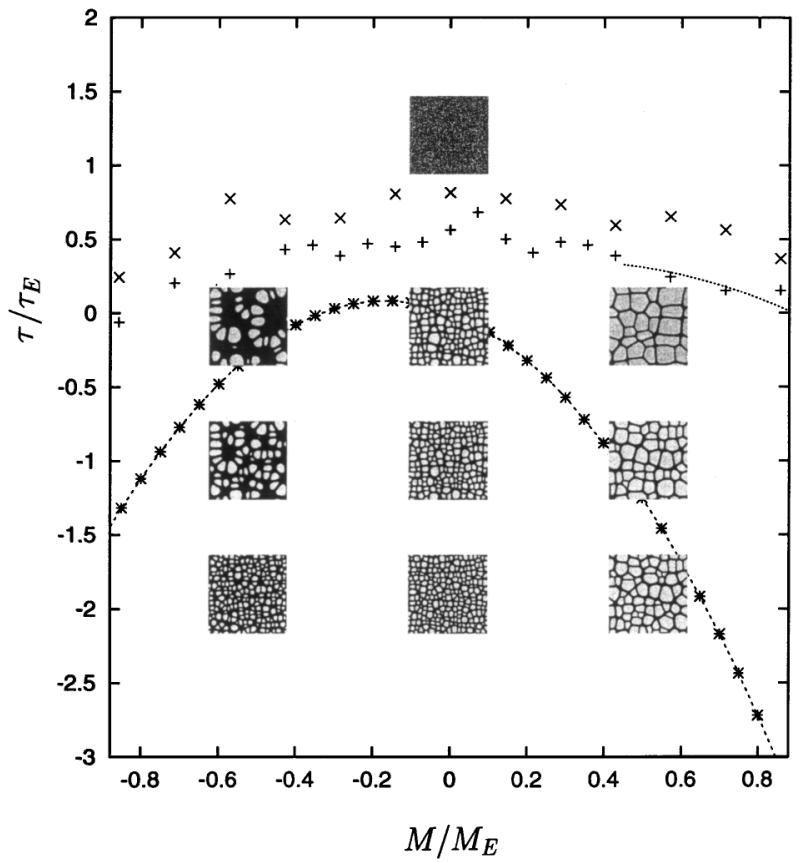

FIG. 3. The phase diagram in the $\tau-M$ (temperaturecomposition) plane under elastic inhomogeneity within isotropic elasticity calculated in two dimensions. The meanings of the data are as follows: +, first order transition points; *, instability points of one-phase states; $\times$, instability points of pinned two-phase states. The points $*$ are on the theoretical spinodal curve (6). The dotted line is obtained from (15) at $\phi_{s}=0.1$. Domain patterns in pinned states are also shown, where the soft regions are written in black.

bilinear dipolar interaction is expressed in terms of $w$ in Eq. (3) as

$$
\frac{1}{k_{B} T} F_{\text {cub }}=\frac{1}{4} \tau_{\text {cub }} \int d \boldsymbol{r} \sum_{i \neq j}\left|\nabla_{i} \nabla_{j} w\right|^{2},
$$

where $\tau_{\text {cub }}=-2 \alpha^{2} \xi_{a} C_{44} / k_{B} T C_{11}^{2}$. If $\tau_{\text {cub }}>0$, the softest directions are [01] and [10] and the domains tend to be rectangular stripes aligned in [10] or [01]. The role of this interaction is simply to orientate the interfaces in these preferred directions [16]. The above form has been used in computer simulations. The total free energy is now the sum of $F$ in Eq. (4) and $F_{\text {cub }}$ in Eq. (16). By setting $\tau_{\text {cub }} / \tau_{E}=0.71$, we examine the phase transition between one-phase states and pinned two-phase states. Figure 4 shows the phase diagram in the $\tau-M$ plane. We can see that the interface orientation is strongly influenced by the elastic anisotropy, but the hysteretic behavior persists at any $M$. Therefore, there is no critical point in the cubic case.

In summary, we have revealed unique phase transition behavior in elastically inhomogeneous alloys, where there is no continuous phase transition and glassy two-phase states are realized even without quenched disorder [17]. We believe that these results hold in both two and three dimensions. In three dimensions they have been confirmed only for $M=0$ in our simulations (not presented here). 


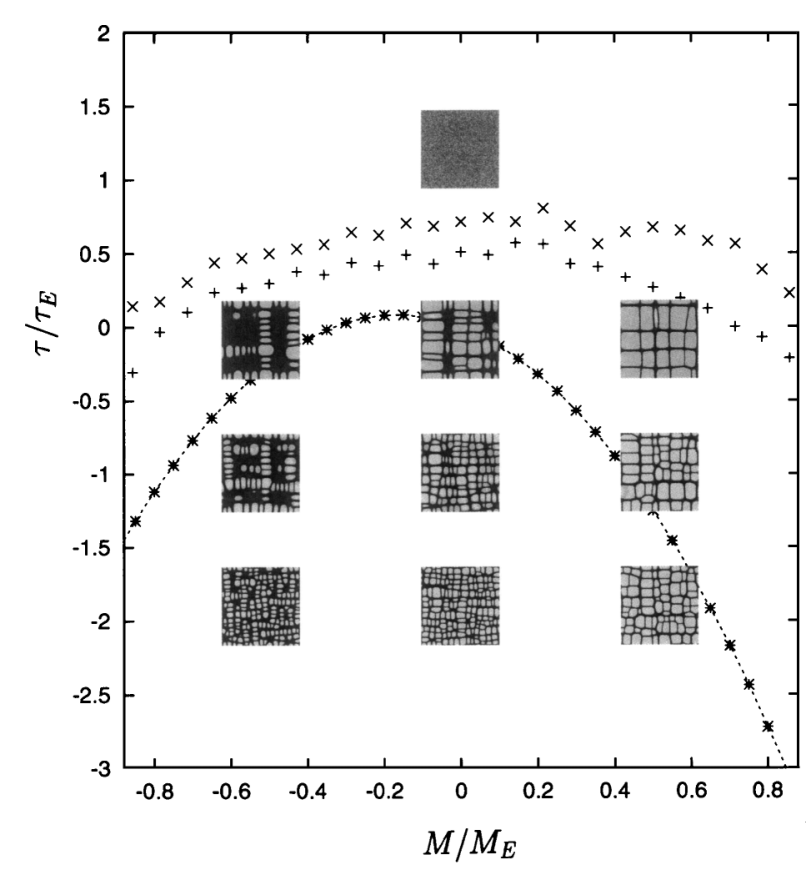

FIG. 4. The phase diagram in the $\tau-M$ plane for elastically inhomogeneous cubic solids. The meanings of the data points are the same as in Fig. 3. Also in this case, there is no critical point.

However, when the volume fraction of the hard component is small, it remains unsettled how the elastic inhomogeneity affects the late stage coarsening. In such cases, shape changes of hard domains will occur when their sizes exceed $R_{E}$ in Eq. (10). But no theory is available on the subsequent phase separation behavior. Finally, we also comment on the effect of elastic inhomogeneity on the phase transition behavior in symmetrical tricritical systems. We assume that the lattice constant and the shear modulus linearly depend on $\psi^{2}$, where $\psi$ is the order parameter [18]. Then the elastic inhomogeneity interaction is of the order of $\psi^{6}$ and is marginal in the tricritical region. Along the first order transition line $R_{E}$ is of the order of $\xi / g_{E}$ and $(\Delta F)_{E} / k_{B} T \sim\left(R_{E} / \xi\right)^{2}$ from Eqs. (10) and (11). Thus, for sufficiently small $g_{E}$, the tricritical point and the critical line do not disappear and glassy two-phase states are realized in unstable regions with domain sizes of the order of $R_{E}(\gg \xi)$.

[1] As a recent review, see P. Fratzl, O. Penrose, and J. L. Lebowitz, J. Stat. Phys. 95, 1429 (1999).

[2] A. G. Khachaturyan, Theory of Structural Transformations in Solids (John Wiley \& Sons, New York, 1983).

[3] J. W. Cahn, Acta Metall. 9, 795 (1961).

[4] J. W. Cahn, Acta Metall. 10, 179 (1962).

[5] A. J. Ardell, R. B. Nicholson, and J.D. Eshelby, Acta Metall. 14, 1295 (1966).

[6] A. Maheshwari and A. J. Ardell, Phys. Rev. Lett. 70, 2305 (1993).

[7] T. Miyazaki and M. Doi, Mater. Sci. Eng. A 110, 175 (1989); T. Miyazaki, M. Doi, and T. Kozakai, Solid State Phenom. 384, 227 (1988).

[8] R. W. Carpenter, Acta Metall. 15, 1567 (1967).

[9] H. A. Calderon, G. Kostorz, Y. Y. Qu, H. J. Dorantes, J. J. Cruz, and J. G. Cabanas-Moreno, Mater. Sci. Eng. A 238, 13 (1997).

[10] A. Ges, O. Rornaro, and H. Palacio, J. Mater. Sci. 32, 3687 (1997).

[11] A. Onuki, J. Phys. Soc. Jpn. 58, 3065 (1989); 58, 3069 (1989).

[12] H. Nishimori and A. Onuki, Phys. Rev. B 42, 980 (1990).

[13] A. Onuki and H. Nishimori, Phys. Rev. B 43, 13649 (1991).

[14] H. Nishimori and A. Onuki, J. Phys. Soc. Jpn. 60, 1208 (1991); Phys. Lett. A 162, 323 (1992).

[15] C. Sagui, D. Orlikowski, A. M. Somoza, and C. Roland, Phys. Rev. E 58, R4092 (1998); D. Orlikowski, C. Sagui, A. M. Somoza, and C. Roland, Phys. Rev. B 59, 8646 (1999).

[16] There can be a concentration dependence in $C_{11}-C_{12}-2 C_{44}$ in cubic solids. This leads to another third order, elastic inhomogeneity interaction, which can strongly affect the domain morphology [15]. In the present work we consider the third order interaction from the inhomogeneity of $C_{44}$ only.

[17] A. Onuki, J. Phys. Soc. Jpn. 68, 5 (1999). At structural phase transitions, pinned two-phase states were shown to exit in a certain temperature interval in the presence of cubic elastic anharmonicity even without quenched disorder.

[18] C. Sagui, A. M. Somoza, and R. C. Desai, Phys. Rev. E 50, 4865 (1994). 